\title{
Bridging the micro and macro for granular media: A computational multi-scale paradigm
}

\author{
J.D. Zhao \& N. Guo \\ Department of Civil and Environmental Engineering, Hong Kong University of Science and Technology, \\ Clearwater Bay, Kowloon, Hong Kong
}

\begin{abstract}
We present a hierarchical multi-scale framework to model geotechnical problems relevant to granular media. The framework employs a rigorous hierarchical coupling between the Finite Element Method (FEM) and the Discrete Element Method (DEM). The FEM is used to discretise the macroscopic geometric domain of a boundary value problem into a FEM mesh. A DEM assembly with memory of its loading history is embedded at each Gauss integration point of the mesh to receive the global deformation from the FEM as input boundary conditions and is solved for the incremental stress-strain relation at the specific material point to advance the FEM computation. The hierarchical framework helps to avoid the phenomenological nature of conventional continuum approaches of constitutive modelling and meanwhile retains the computational efficiency of the FEM in solving large-scale Boundary Value Problems (BVPs). By virtue of its hierarchical structure, the predictive power of the proposed method can be further unleashed with proper implementation of parallel computing techniques. By corroborating the rich information obtained from the particle level with the macroscopically observed responses, the framework helps to shed light on a cross-scale understanding of granular media. We demonstrate the predictive capability of the proposed framework by simulations of shear localisations in monotonic biaxial compression and cavity inflation in a thick-walled cylinder, as well as liquefaction and cyclic mobility in cyclic simple shear tests.
\end{abstract}

\section{INTRODUCTION}

Granular materials are multi-scale by nature. When subject to shear, a granular material may exhibit complicated macroscopic behaviours that are notoriously difficult to characterise, such as state dependency, strength anisotropy, strain localisation, non-coaxiality, solid-flow phase transition (e.g., liquefaction) and critical state (Guo \& Zhao 2013b, Zhao \& Guo 2013). These macroscopic responses reflects highly complicated microstructural mechanisms established and evolved at the granular particle level during the loading process. While a granular medium has long been treated by continuum mechanics, it becomes increasingly clear now that a better understanding can only be achieved with the aid of effective bridging approaches linking the micro to the macro scales of the material. Various homogenisation techniques have been developed in material sciences to link different length scales of a material for integrated characterisation of the material behaviour. They are targeted at designing engineered or new materials with identifiable microstructure to achieve optimal engineering performance of various purposes. However, several intrinsic properties associated with granular media exclude the possibility of deriving material properties and predicting the material responses directly from the particle scale through analytical homogenisation methods as the material science branch does.
There are two outstanding ones: (1) The lacking of periodic microstructure in a granular material prevents a general homogenisation method working effectively, which is due mainly to the great randomness and heterogeneity within a granular system; (2) The behaviour of a granular material is generally statedependent and loading-path specific. It is thus difficult to identify a once-for-all microstructure from which the macroscopic properties of the material can be derived via homogenisation. To resolve the bridging issue, a computational multi-scale modelling approach appears to be a viable (if not the only) option. In this study, we propose a hierarchical multi-scale framework on micro-macro bridging for granular media. The framework employs a rigorous hierarchical coupling between the finite element method (FEM) and the discrete element method (DEM) to solve boundary value problems relevant to granular media. The study is in line with a number of previous attempts on this topic (Meier et al. 2008, Meier et al. 2009, Andrade et al. 2011, Nitka et al. 2011, Guo and Zhao 2013a).

\section{METHODOLOGY AND FORMULATION}

\subsection{Hierarchical multiscale modelling approach}

The multiscale framework employs a rigorous hierarchical coupling between the finite element method 


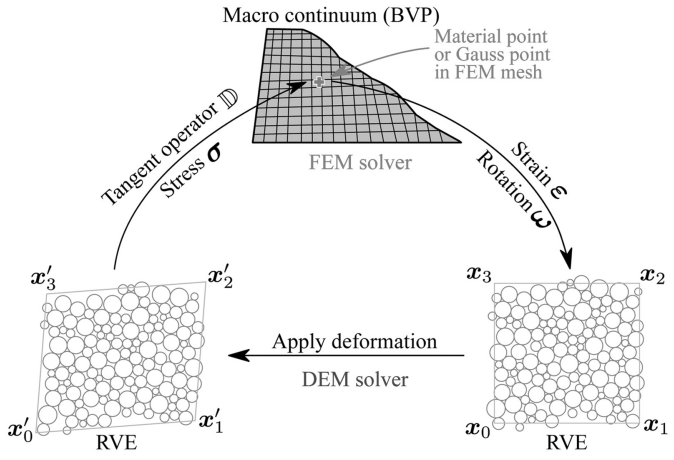

Figure 1. A hierarchical multiscale modelling framework for granular media based on coupled FEM/DEM.

(FEM) and the discrete element method (DEM) which is schematically shown in Fig. 1. To solve a boundary value problem, the macroscopic geometric domain is first discretized into a FEM mesh. A DEM assembly is then embedded at each Gauss integration point of the mesh serving as a local Representative Volume Element (RVE). At each load step, the RVE takes its memory of the past loading history as initial conditions and receives the global deformation from the FEM at the specific Gauss point as input boundary conditions. It is solved to derive the local incremental stress-strain relation (e.g., stress and tangential stiffness matrix) required for advancing the global FEM computation. To be more specific, the FEM is used to solve the following equation system for the discretised domain:

$$
\boldsymbol{K u}=\boldsymbol{f}
$$

where $\boldsymbol{K}, \boldsymbol{u}$ and $\boldsymbol{f}$ are the stiffness matrix, the unknown displacement vector at the FEM nodes, and the nodal force vector lumped from the applied boundary traction, respectively. Since $\boldsymbol{K}$ is generally dependent on the state parameters and loading history for a granular medium, linearisation of the solution and NewtonRaphson iterative method are commonly required. In doing so, the tangent operator $\boldsymbol{K}_{t}$ needs to properly evaluated:

$$
\boldsymbol{K}_{t}=\int_{\Omega} \boldsymbol{B}^{T} \boldsymbol{D} \boldsymbol{B} d V
$$

where $\boldsymbol{B}$ is the deformation matrix (i.e. gradient of the shape function), and $\boldsymbol{D}$ is the matrix form of the rank four tangent operator tensor. During each Newton-Raphson iteration, both $\boldsymbol{K}_{t}$ and $\boldsymbol{\sigma}$ are updated to minimise the following residual force $\boldsymbol{R}$ to find a converged solution

$$
\boldsymbol{R}=\int_{\Omega} \boldsymbol{B}^{T} \boldsymbol{\sigma} d V-\boldsymbol{f}
$$

Instead of making phenomenological assumptions for the instantaneous tangent modulus $\boldsymbol{D}^{e p}$ in Equation 2 to assemble $\boldsymbol{K}_{t}$ as conventional continuumbased constitutive modelling approaches do (e.g., by assuming an elasto-plastic stiffness matrix in classic plasticity theory), the coupled FEM/DEM multi scale approach determines the two quantities from the embedded discrete element assembly at each Gauss point. In doing so each DEM packing receives the boundary condition (deformation) by interpolation of the FEM solution (displacement). Upon reaching a solution, each DEM is then homogenised to derive the stress and tangent operator at the material point which are transferred back to the FEM solver to update the solution. The Cauchy stress tensor is homogenised by the following expression

$\boldsymbol{\sigma}=\frac{1}{V} \sum_{N_{c}} \boldsymbol{d}^{c} \otimes \boldsymbol{f}^{c}$

where $V$ is the total volume of the DEM assembly, $N_{c}$ is the number of contacts within the volume, $\boldsymbol{f}^{c}$ and $\boldsymbol{d}^{c}$ are the contact force vector and the branch vector connecting the centres of the two contacted particles, respectively.

In deriving the tangent operator, we use the following bulk elastic modulus homogenised from the DEM assembly as a first coarse estimation of the the tangent operator:

$$
\boldsymbol{D}^{e}=\frac{1}{V} \sum_{N_{c}}\left(k_{n} \boldsymbol{n}^{c} \otimes \boldsymbol{d}^{c} \otimes \boldsymbol{n}^{c} \otimes \boldsymbol{d}^{c}+k_{t} \boldsymbol{t}^{c} \otimes \boldsymbol{d}^{c} \otimes \boldsymbol{t}^{c} \otimes \boldsymbol{d}^{c}\right)
$$

where $k_{n}$ and $k_{t}$ are the equivalent normal and tangential stiffnesses describing the contact law of the particles, $\boldsymbol{n}^{c}$ and $\boldsymbol{t}^{c}$ are unit vectors in the outward normal and tangential directions of a contact, respectively. ' $\otimes$ ' denotes the dyadic product of two vectors. In conduction with an iterative scheme, it works more efficient in deriving the tangent operator in comparison with the alternative perturbation-based method (Meier et al. 2009, Nitka et al. 2011, Guo and Zhao 2014).

\subsection{Solution procedure}

The solution procedure to the hierarchical multi-scale modeling approach is summarized as follows:

I. Discretise the macro domain by a suitable FEM mesh and embed a DEM assembly with appropriate initial state at each Gauss point of the mesh as a RVE.

II. Apply one global load step imposed by the boundary traction of the FEM domain.

(a) Determine the current tangent operator using Eq. 5 for each RVE.

(b) Assemble the global tangent matrix using Eq. 2 and compute a trial solution of displacement $\boldsymbol{u}$ by solving Eq. 1 with FEM.

(c) Interpolate the deformation $\nabla \boldsymbol{u}$ at each Gauss point of the FEM mesh and run the DEM simulation for the corresponding RVE by accounting for its initial state and using $\nabla \boldsymbol{u}$ as the DEM boundary conditions. 

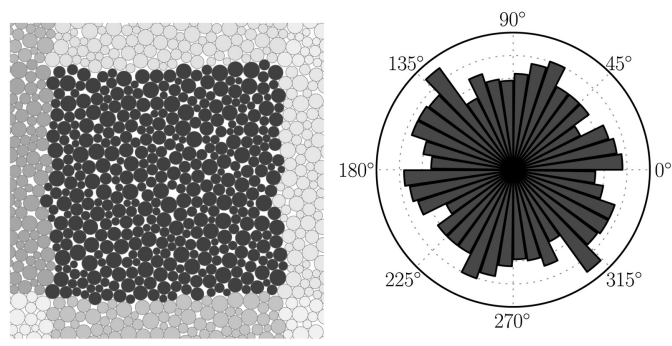

Figure 2. The calibrated RVE comprised of 400 polydisperse spheres with periodic boundary produces isotropic contact normal distribution under isotropic compression.

(d) Derive the updated total stress from Eq. 4 for each RVE and use them to evaluate the residual by Eq. 3 for the FEM domain.

(e) Repeat the above steps from (a) to (d) until convergence is reached.

(f) Save the converged solution of each RVE as its new initial state for next step and finish the current load step.

III. Advance to next load step and repeat Step II.

\subsection{Benchmark and calibration}

The proposed hierarchical multi-scale approach has been successfully implemented by two open source codes for FEM and DEM (Guo \& Zhao 2014). A simple linear force-displacement contact law in conjunction with Coulomb's friction is employed in the DEM code to describe the stick-slip inter particle contact. Polydisperse particles with radii ranging from $3 \mathrm{~mm}$ to $7 \mathrm{~mm}\left(r_{\text {mean }}=5 \mathrm{~mm}\right)$ are adopted to generate each DEM assembly. Quasi-static solutions are solved at each load step for each RVE. To calibrate the size of the RVE (e.g., particle number), different RVEs with different particle numbers subject to isotropic compression have been compared and the resultant contact normal distribution is examined. A typical RVE containing 400 particles and with periodic boundary is found to provide largely isotropic contact normal distribution and meanwhile offer reasonable computational efficiency (see Fig. 2), which will be adopted for all the simulations in the sequel.

A single-element shear test based on first-order four-node quadrilateral element has been used to benchmark the multi-scale method. All RVEs at the four Gauss points possess identical initial conditions, which leads to a uniform sample for the FEM solution. The global response of the single element test is compared against that obtained from a pure DEM test based on the RVE in Fig. 3. The global vertical stress $\sigma_{11}$ is calculated from the resultant force exerted on the top boundary and $\sigma_{00}$ from the resultant lateral force. The axial strain $\epsilon_{11}$ and the volumetric strain $\epsilon_{v}$ are calculated from the overall deformation of the element. Fig. 3 shows that the multi-scale modelling produced nearly identical responses with the pure DEM simulation for the single element test, which indicates
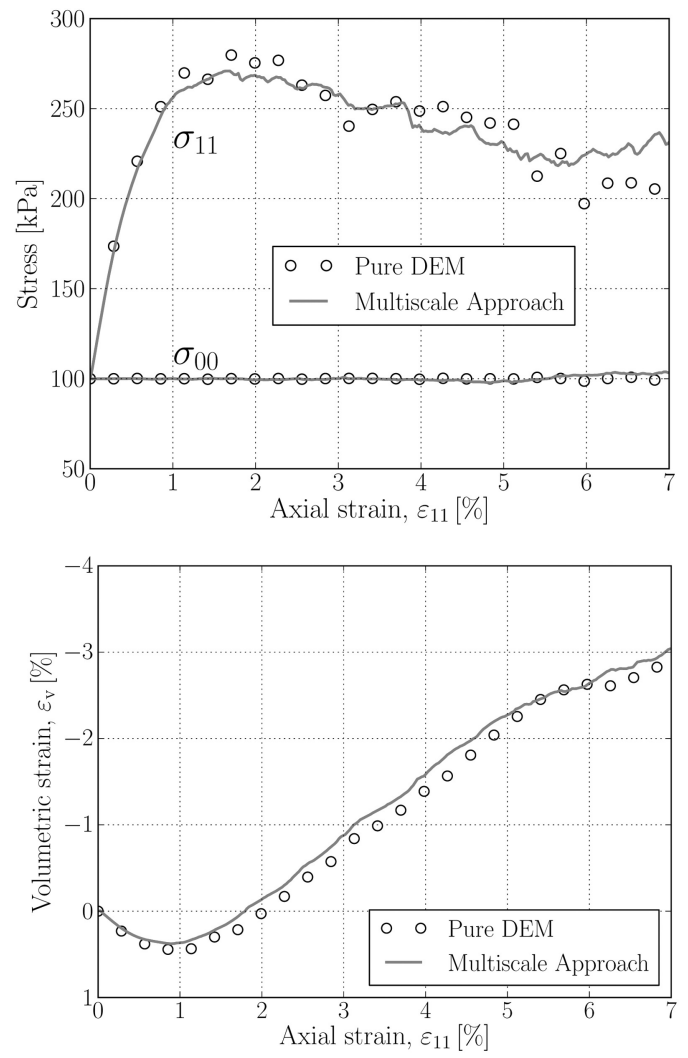

Figure 3. Comparison of the macroscopic responses of the single-element test by the multiscale method and by a pure DEM test of RVE size.

that the hierarchical multi-scale modelling approach is able to faithfully reproduce the typical behaviour of granular media.

Meanwhile, in our hierarchical multi-scale framework, the computation of DEM packing attached to each Gauss point is independent with one another, which makes it ideal for parallelisation of simulations. An effective parallel computing technique has been implemented in our multis-cale code and has been used for all the subsequent simulations with a cluster at HKUST. The scalability and efficiency of the paralleled code has been found rather satisfactory (Guo \& Zhao 2014).

\section{DEMONSTRATIVE EXAMPLES}

\subsection{Monotonic biaxial compression on sand}

We first apply the hierarchical multiscale approach to predicting the response of a sand sample subject to monotonic biaxial compression. The model setup and boundary conditions are shown in Fig. 4 (left) where the FEM mesh consists of four-node linear quadrilateral elements. Fig. 4 (right) presents the global stress-strain response measured from the boundary 

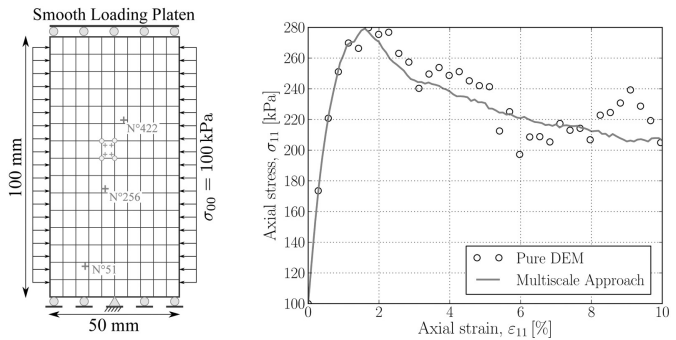

Figure 4. Multiscale modelling of monotonic biaxial compression on sand: (left) model setup; (right) global stress strain relationship for the sand sample (calculated from the resultants at the boundary).

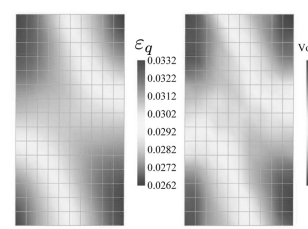

(a) $\varepsilon_{11}=1.6 \%$ (peak)

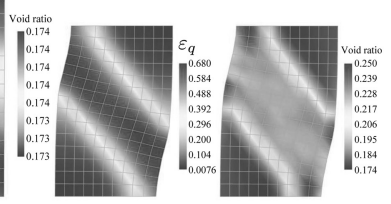

(b) $\varepsilon_{11}=10 \%$ (final)
Figure 5. Contours of the accumulated deviatoric strain and void ratio showing strain localisation in biaxial compression of sand.

reaction forces and displacements. A comparison case is also presented for the pure DEM simulation of the biaxial shear on a RVE size sample. Notably, the global response by the multiscale modelling approach bears great similarity to the RVE response, in particular for the pre-peak stress stage where the material behaviour is relatively elastic. While the post-peak response of the DEM test shows moderate fluctuations, the multi-scale model results are relatively smooth.

Our multi-scale simulation captures the phenomenon of strain localisation that is commonly found in laboratory biaxial shear tests (see Fig. 5). This is interesting by the following reason. Our biaxial shear simulation has been set up with smooth and symmetric boundary conditions and loading. The initial states for all RVE packings in the FEM mesh are identical and hence the entire sample is homogeneous too. Under such symmetric/homogeneous conditions, conventional continuum-model-based FEM approaches are generally unable to capture the strain localisation unless certain artificial imperfection(s) or random distributed local properties are added to the sample to break the symmetry and trigger strain localisation. In our multi-scale modelling of the problem, though the RVEs are identical and homogeneous in the FEM mesh, there is small but observable initial anisotropy present in the initial RVE packing (see Fig. 2), which may serve as the symmetry breaker to trigger the occurrence in our mutliscale modelling. Similar opinion has been discussed by Gao \& Zhao (2013) based on continuum modelling. The localised region observed from Fig. 5 concentrates in the most dilative portion of the sample with large void ratio, which is consistent with

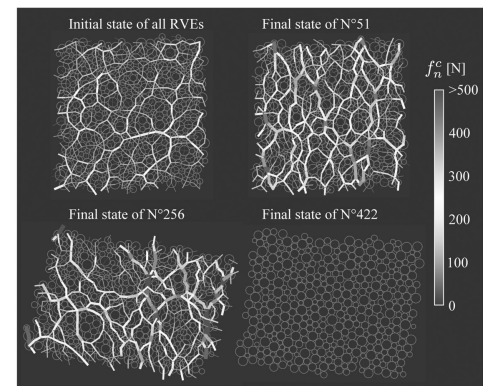

(a)

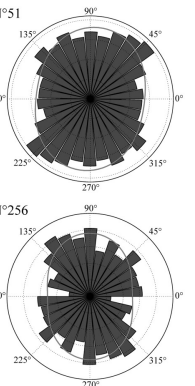

(b)
Figure 6. (a) The structure and force chains of the local initial and deformed RVEs and (b) the corresponding distributions of the contact normals in $\mathrm{N}^{\circ} 51$ and $\mathrm{N}^{\circ} 256$. The smooth red curves in (b) are the second-order Fourier approximations.

experimental observations. The localised band of void ratio is found generally wider than that of the deviatoric strain.

A major advantage of the multi-scale framework lies in the rich micro-scale information it can provide when solving an engineering scale problem. This may greatly facilitate a better correlation and understanding of the macroscopic observations. Shown in Fig. 6 is a comparison of the contact force network for the RVE packings at the three Gauss points indicated in Fig. 4 at the initial and final loading states. All RVEs have the same initial isotropic condition without apparent preferably orientated strong force chains. While the DEM packing at $\mathrm{N}^{\circ} 51$ does not experience much deformation, several distinct strong force chains are observed aligning parallel to the vertical shear direction. In contrast, the RVE at $\mathrm{N}^{\circ} 256$ experiences severe shear deformation which results in a highly heterogeneous internal structure and more concentrated penetrating force chains aligning to the vertical. The packing at $\mathrm{N}^{\circ} 422$ also deforms noticeably, but it becomes rather dilute due to remarkable volumetric expansion. The deformation gradients at $\mathrm{N}^{\circ} 256$ and $\mathrm{N}^{\circ} 422$ are consistent with the global shear band inclination. An inspection of the contact normal distribution (shown in Fig. 6) further confirms that the major principal directions for both $\mathrm{N}^{\circ} 51$ and $\mathrm{N}^{\circ} 256$ are close to the vertical shear direction.

\subsection{Cyclic simple shear test}

The multiscale modelling approach has also been applied to simulating the hysteresis behaviour of sand subject to cyclic load. Shown in Fig. 7 is a FEM mesh and boundary conditions for a sand sample subject to maximum shear stress controlled cyclic simple shear. The initial packing is chosen to be relatively loose for all RVEs (different than the other examples where dense packing is used). The global stress-strain response and loading path are shown in Fig. 8, indicating a typical hysteresis behaviour of sand observed in laboratory tests. The contour of the void ratio in Fig. 9 indicates that the deformation in the sample 


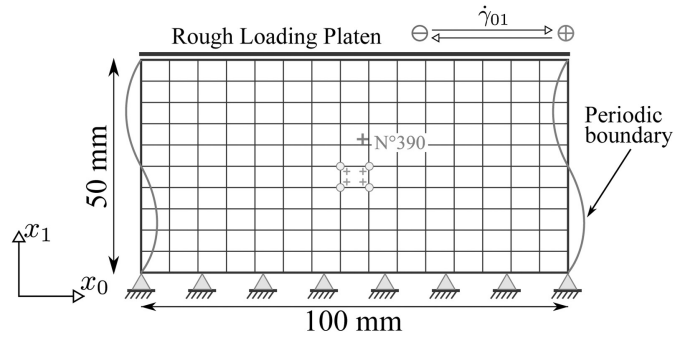

Figure 7. Discretization $(15 \times 10$ elements $)$ and boundary condition of the specimen for cyclic simple shear. The $\mathrm{N}^{\circ} 390$ Gauss point will be used for local analyses.

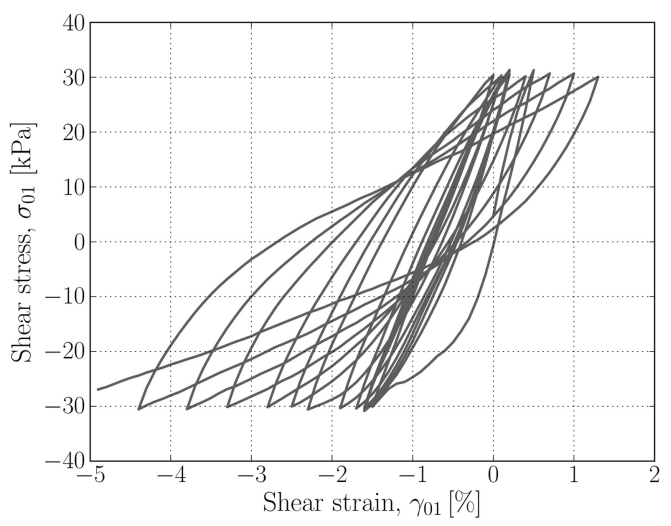

(a) Shear stress-strain relation

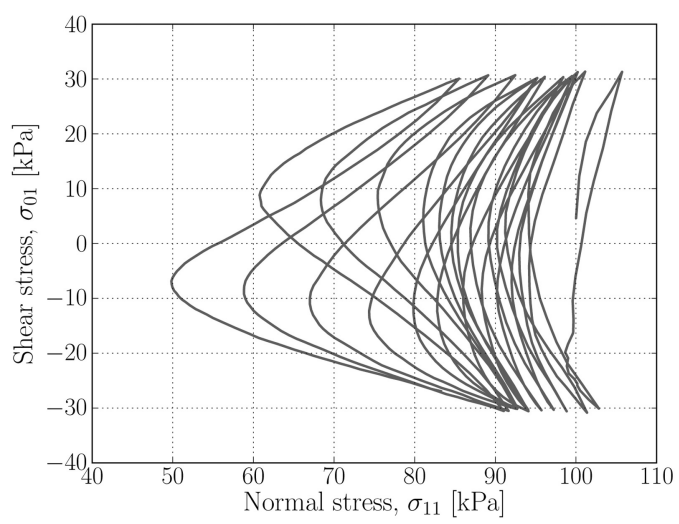

(b) Normal-shear stress relation

Figure 8. Global responses of the cyclic simple shear test under maximum shear stress control $\left|\sigma_{01}\right|=30 \mathrm{kPa}$.

is relatively uniform. The RVE at $\mathrm{N}^{\circ} 390$ shows several obvious strong force chains aligning along the diagonal direction of the packing. As the accumulated shear strain becomes larger, it is evident that cyclic mobility occurs for this sample. Not presenting here due to length limitation, we have also investigated a comparison case where the same sample is subject to constant-volume maximum shear strain controlled cyclic shear wherein we found liquefaction

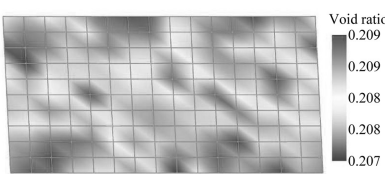

(a)

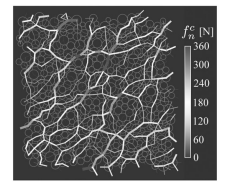

(b)
Figure 9. (a) Contour of void ratio at the final state after the maximum shear stress controlled cyclic loading and (b) the structure and the force chains in RVE at $N^{\circ} 390$.

occurs. At the liquified material points, the contact network of the RVE become too week to form effective percolating force chains to sustain the external shear.

\subsection{Cavity inflation in thick-walled hollow cylinder}

The multi-scale approach has also been applied to modelling the cavity inflation in thick-walled cylinder as treated experimentally by Alsiny et al. (1992). Thick-walled hollow cylinder inflation experiments are commonly performed towards a better understand the soil behaviour under passive loading conditions with simultaneous extension in a plane perpendicular to the loading direction. A quarter of a whole thickwalled hollow cylinder is simulated, with an identical geometry of that in Alsiny et al. (1992) for the cavity and outer surface of the cylinder: $r_{c}=15 \mathrm{~mm}$ and $r_{o}=$ $150 \mathrm{~mm}$. Due to symmetry, the displacement of the left boundary is fixed and the vertical displacement of the bottom boundary is fixed (see Fig. 10a). Eight-node elements were used in the study. The outer boundary is prescribed by a constant pressure $p_{0}=100 \mathrm{kPa}$. Different than the inflation pressure boundary in Alsiny et al. (1992), a Neumann boundary with gradually increased displacement $u_{c}$ is applied to the inner surface until $u_{c}=10 \mathrm{~mm}$. Fig. $10 \mathrm{~b}$ depicts the inflation-pressure response (the differential cavity pressure is defined by the pressure difference on the inner and outer cylinder walls), which shows a clear softening portion of the curve. Our simulation shows that the occurrence of shear localisation in the cylinder was initiated shortly beyond the peak inflation pressure, which is consistent with the experimental finding by Alsiny et al. (1992). Since our simulation uses Neumann boundary at the cavity surface, no diffuse deformation mode has been detected.

Fig. 11 presents the localised distributions of shear strain, void ratio and average rotation (obtained from the RVE) within the cylinder at the end of the inflation. The three contours show apparently good correlations. Within the shear bands the soil is severely sheared, highly dilated (initial void ratio is 0.177 ) and experiences considerable rotation of soil particles. The shear bands originated from the cavity surface form cross-shaped patterns which have been observed in laboratory tests. The soil particles within the bands of different orientation have been rotated in totally different direction. Other than those close to the cavity, there are two less developed, notable shear bands touching 


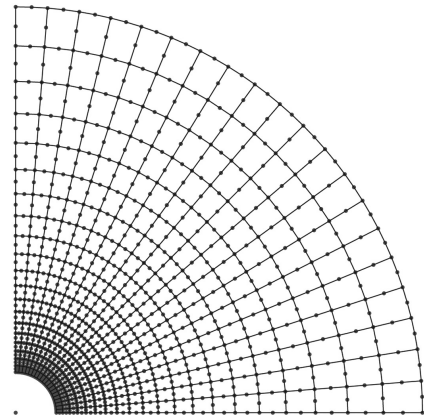

(a)

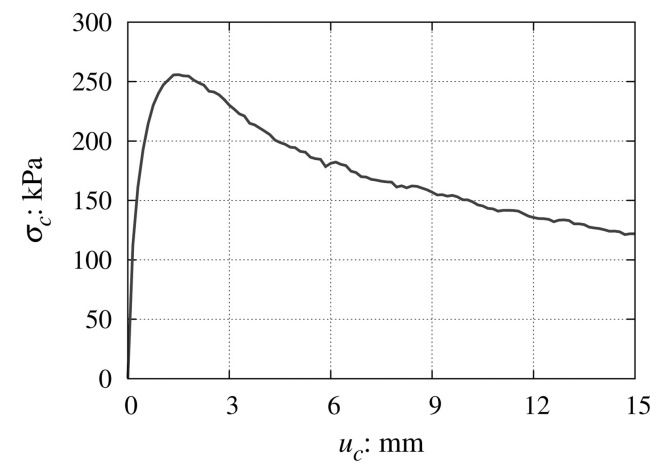

(b)

Figure 10. Multiscale simulation of cavity inflation of a thick-walled hollow cylinder in sand: (a) mesh; (b) pressure-inflation curve.

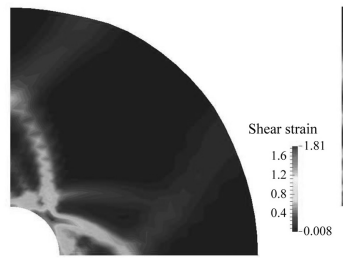

(a)

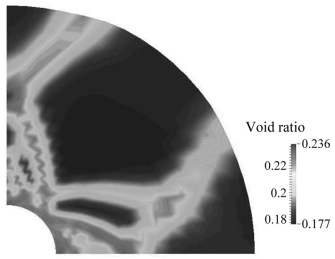

(b)

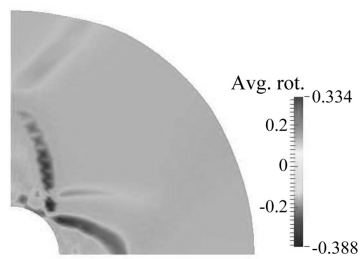

(c)

Figure 11. Localised distributions for (a) shear strain, (b) void ratio and (c) average rotation in the cylinder.

the outer surface of the cylinder. They are however not originated directly from the cavity surface, but from the two fixed boundaries. They appear to be reflections of the two major shear bands initiated from the cavity surface. Further investigation based on a full cylinder rather than its quarter will be carried out to see if this is the case. The shear band orientation will also be analysed based on more refined model of the problem in the future.

\section{CONCLUSIONS}

A hierarchical multi-scale framework has been developed to bridge the micro and macro behaviours of granular media for practical modelling of engineering scale problems. Based on a rigorous coupling between FEM and DEM, it circumvents the phenomenological assumptions commonly required in continuum constitutive modelling and meanwhile retains a good predictive capability on solving practical problems which purely micromechanics-based approaches are inherently restrained to solve. The framework has been benchmarked, calibrated and enhanced with parallel computing techniques. Its predictive capability has been demonstrated with three illustrative examples, including monotonic biaxial compression test, cyclic simple shear tests and cavity inflation in thick-walled hollow cylinder.

\section{REFERENCES}

Alsiny, A., I. Vardoulakis, \& A. Drescher (1992). Deformation localisation in cavity inflation experiments on dry sand. Géotechnique 42(3): 395-410.

Andrade, J. E., C. F. Avila, S. A. Hall, N. Lenoir, \& G. Viggiani (2011). Multiscale modeling and characterization of granular matter: from grain kinematics to continuum mechanics. Journal of the Mechanics and Physics of Solids 59: 237-250.

Gao, Z. \& J. Zhao (2013). Strain localization and fabric evolution in sand. International Journal of Solids and Structures 50: 3634-3648.

Guo, N. \& J. Zhao (2013a). A hierarchical model for crossscale simulation of granular media. In AIP Conference Proceedings, Volume 1542, pp. 1222-1225.

Guo, N. \& J. Zhao (2013b). The signature of shear-induced anisotropy in granular media. Computers and Geotechnics 47: 1-15.

Guo, N. \& J. Zhao (2014). A coupled FEM/DEM approach for hierarchical multiscale modelling of granular media. International Journal for Numerical Methods in Engineering In press. DOI: 10.1002/nme.4702.

Meier, H., P. Steinmann, \& E. Kuhl (2009). On the multiscale computation of confined granular media. In J. Eberhardsteiner, C. Hellmich, H. Mang, and J. Périaux (Eds.), ECCOMAS Multidisciplinary Jubilee Symposium, Volume 14 of Computational Methods in Applied Sciences, pp. 121-133. Springer Netherlands.

Meier, H. A., P. Steinmann, \& E. Kuhl (2008). Towards multiscale computation of confined granular mediacontact forces, stresses and tangent operators. Technische Mechanik 28(1): 32-42.

Nitka, M., G. Combe, C. Dascalu, \& J. Desrues (2011). Two-scale modeling of granular materials: a DEM-FEM approach. Granular Matter 13: 277-281.

Zhao, J. \& N. Guo (2013). Unique critical state characteristics in granular media considering fabric anisotropy. Géotechnique 63(8): 695-704. 